\title{
A Gaussian Mixture PHD filter for Extended Target Tracking
}

\author{
Karl Granström, Christian Lundquist, Umut Orguner \\ Division of Automatic Control, Department of Electrical Engineering \\ Linköping University, Linköping, Sweden \\ \{karl,lundquist,umut\}@isy.liu.se
}

\begin{abstract}
In extended target tracking, targets potentially produce more than one measurement per time step. Multiple extended targets are therefore usually hard to track, due to the resulting complex data association. The main contribution of this paper is the implementation of a Probability Hypothesis Density (PHD) filter for tracking of multiple extended targets. A general modification of the PHD filter to handle extended targets has been presented recently by Mahler, and the novelty in this work lies in the realisation of a Gaussian mixture PHD filter for extended targets. Furthermore, we propose a method to easily partition the measurements into a number of subsets, each of which is supposed to contain measurements that all stem from the same source. The method is illustrated in simulation examples, and the advantage of the implemented extended target PHD filter is shown in a comparison with a standard PHD filter.
\end{abstract}

Keywords: multi target tracking, filtering, estimation, extended targets, probability hypothesis density, Gaussian mixture.

\section{Introduction}

The purpose of multi target tracking is to detect, track and identify targets from sequences of noisy, possibly cluttered, measurements. In most applications, it is assumed that each target produces at most one measurement per time step. This is true for some cases, e.g. in radar applications when the distance between the target and the sensor is large. In other cases however, the distance between target and sensor, or the size of the target, may be such that multiple resolution cells of the sensor are occupied by the target. This is the case with e.g. image sensors. Targets that potentially give rise to more than one measurement are denoted as extended.

Gilholm and Salmond [3] presented an approach for tracking extended targets under the assumption that the number of recieved target measurements in each time step is Poisson distributed. They show an ex- ample where they track point targets which may generate more than one measurement, and an example where they track objects that have a 1-D extension (infinitely thin stick of length $l$ ). In [2] a measurement model was suggested which is an inhomogeneous Poisson point process. At each time step, a Poisson distributed random number of measurements are generated, distributed around the target. This measurement model can be understood to imply that the extended target is sufficiently far away from the sensor for its measurements to resemble a cluster of points, rather than a geometrically structured ensemble. A similar approach is taken in [1], where Track Before Detect theory is used to track a point target with a 1-D extent.

Using Finite Set Statistics (FISST), Mahler has presented a rigorous framework for target tracking employing the so called Probability Hypothesis Density (PHD) filter [4]. A random finite set (RFS) is a set with a random number of elements and where each element in the set is a random variable. In the PHD filter the targets and measurements are treated as RFS, which allows the problem of estimating multiple targets in clutter and uncertain associations to be cast in a Bayesian filtering framework [4]. An implementation of a linear Gaussian PHD-filter was presented in [7]. There the PHD filter is approximated with a mixture of Gaussians, hence the realization is called Gaussian Mixture-PHD (GM-PHD) filter. In the recent work [6], Mahler presented an extension of the PHD filter to also handle extended targets of the type presented in [2].

In this paper, we extend the work in $[2,6,7]$, and present a GM-PHD-filter for extended target tracking. To the best of our knowledge, such a filter has not been presented before. We present and define the target tracking problem in the Section 2. The dynamic and measurement models are both assumed to be linear Gaussian, and the number of measurements generated by each target in each time step is assumed to be random samples from Poisson distributed variables. 
The number of clutters generated is also assumed to be Poisson distributed. For the measurement update step of the extended target GM-PHD filter, different partitions of the set of measurements have to be considered. In Section 3 a simple method for finding measurement set partitions is presented. The underlying intuition behind the paritioning method is that measurements generated by the same target will be spatially close. The measurement likelihood is presented in Section 4, where the suggested GM-PHD filter for extended target tracking is also derived. In Section 5 results from simulations are presented, both for single and multiple extended target tracking. Finally, Section 6 contains conclusions and thoughts on future work.

\section{The Target Tracking Problem}

The aim of this work is to estimate an RFS of targets $\mathbf{X}_{k}=\left\{\mathbf{x}_{k}^{(i)}\right\}_{i=1}^{N_{x, k}}$, given a RFS of measurements $\mathbf{Z}_{k}=$ $\left\{\mathbf{z}_{k}^{(i)}\right\}_{i=1}^{N_{z, k}}$, for discrete time instants $k=1, \ldots, K$. Each target $\mathbf{x}_{k}^{(i)}$ in the RFS $\mathbf{X}_{k}$ is assumed to be modelled using a linear Gaussian dynamical model,

$$
\mathbf{x}_{k+1}^{(i)}=F_{k} \mathbf{x}_{k}^{(i)}+G_{k} \mathbf{w}_{k}^{(i)}
$$

where $\mathbf{w}_{k}^{(i)}$ is Gaussian white noise with covariance $Q_{k}^{(i)}$. Each measurement is generated according to a linear Gaussian model,

$$
\mathbf{z}_{k}^{(j)}=H_{k} \mathbf{x}_{k}^{(i)}+\mathbf{e}_{k}^{(j)}
$$

where $\mathbf{e}_{k}^{(j)}$ is white Gaussian noise with covariance $R_{k}^{(j)}$. Note that there is no known association between the targets and the measurements.

In previous work, extended targets have often been modelled as targets having a spatial extension or shape, however the problem is sometimes simplified by assuming that the targets are points [3]. In reality however, all targets have a spatial extension or shape. Whether shape parameters should be included in the target state vector largely depends on the size of the target compared to the sensor resolution. Airplanes that are tracked by radar typically give rise to at most one measurement and can often be efficiently modelled as points, while vehicles tracked by laser range sensors typically give rise to multiple measurements from which the shape and size of the vehicle can be inferred. To the best of our knowledge, exactly what is meant by extended target has not been definitely defined in the target tracking literature. Therefore we propose the following definition:

Definition: Extended targets are targets that potentially give rise to more than one measurement per time step.

In this work, the number of measurements generated by each target at each time step, denoted $N_{m, k}^{(i)}$, is a Poisson distributed random variable with rate $\beta_{D}$ measurements per scan, thus the probability of generating at least one measurement is $1-e^{-\beta_{D}}$. A target is detected with probability $p_{D}$, giving the effective probability of detection

$$
p_{D, \mathrm{eff}}=\left(1-e^{-\beta_{D}}\right) p_{D} .
$$

At each time step, clutter measurements are also generated. The number of clutters generated, $N_{k}^{c}$, is a Poisson distributed random variable with rate $\beta_{F A}$ clutter measurements per surveillance volume per scan. Thus, if the surveillance volume is $V_{s}$, the mean value for the Poisson variable $N_{k}^{c}$ is $\beta_{F A} V_{s}$ clutter measurements per scan. The spatial distribution of the clutter measurements is uniform over the surveillance volume.

For example, we have at time $k$ one target and $N_{m, k}^{1}=2$ generated measurements $\mathbf{z}_{k}^{(A)}$ and $\mathbf{z}_{k}^{(B)}$, and $N_{c, k}=1$ clutter measurement $\mathbf{z}_{k}^{(C)}$. Then, at time $k$, the set of measurements obtained by the sensor is

$$
\mathbf{Z}_{k}=\left\{\mathbf{z}_{k}^{(1)}, \mathbf{z}_{k}^{(2)}, \mathbf{z}_{k}^{(3)}\right\}
$$

Note that the true nature of the measurements, i.e. clutter or generated by a target, is not known to the tracking filter, hence the different indexing $A, B, C$ and $1,2,3$. Let $\mathbf{Z}_{1: k}$ denote the set of measurement sets from time 1 to time $k$.

A track with cluttered measurements is shown in Figure 1 , and the number of obtained measurements by the sensor at each time step is shown in Figure 2.

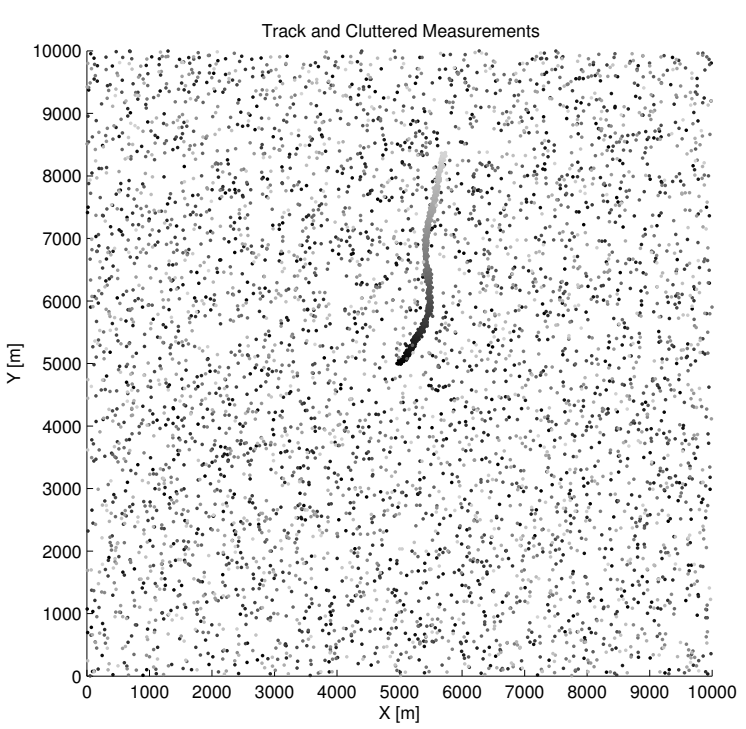

Figure 1: The example shows both target generated measurements and clutter. A Grayscale is used to denote different time steps.

\section{Partitioning the Measurement Set}

An integral part of extended target tracking with the PHD filter is the partitioning of the set of measurements [6]. A partition $\mathrm{p}$ is defined as a division of 


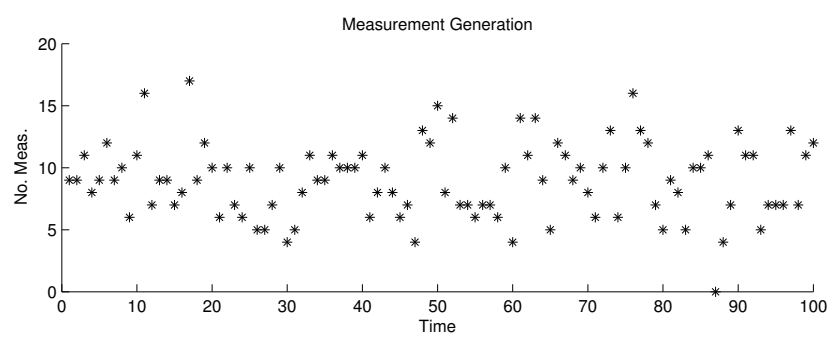

Figure 2: Number of true target measurements generated for the example in Figure 1, i.e. clutter is not included. The measurement per scan rate is $\beta_{D}=10$.

the set of measurements $\mathbf{Z}$ into subsets, called cells $W$. The partitioning is important, since more than one measurement can stem from the same target. Let us exemplify the partitioning principle with a measurement set containing three individual measurements, $\mathbf{Z}_{k}=\left\{\mathbf{z}_{k}^{(1)}, \mathbf{z}_{k}^{(2)}, \mathbf{z}_{k}^{(3)}\right\}$. This set can be partitioned in the following ways [6]:

$$
\begin{aligned}
& \mathrm{p}_{1}: W_{1}^{1}=\left\{\mathbf{z}_{k}^{(1)}, \mathbf{z}_{k}^{(2)}, \mathbf{z}_{k}^{(3)}\right\}, \\
& \mathrm{p}_{2}: W_{1}^{2}=\left\{\mathbf{z}_{k}^{(1)}, \mathbf{z}_{k}^{(2)}\right\}, W_{2}^{2}=\left\{\mathbf{z}_{k}^{(3)}\right\}, \\
& \mathrm{p}_{3}: W_{1}^{3}=\left\{\mathbf{z}_{k}^{(1)}, \mathbf{z}_{k}^{(3)}\right\}, W_{2}^{3}=\left\{\mathbf{z}_{k}^{(2)}\right\}, \\
& \mathrm{p}_{4}: W_{1}^{4}=\left\{\mathbf{z}_{k}^{(2)}, \mathbf{z}_{k}^{(3)}\right\}, W_{2}^{4}=\left\{\mathbf{z}_{k}^{(1)}\right\}, \\
& \mathrm{p}_{5}: W_{1}^{5}=\left\{\mathbf{z}_{k}^{(1)}\right\}, W_{2}^{5}=\left\{\mathbf{z}_{k}^{(2)}\right\}, W_{3}^{5}=\left\{\mathbf{z}_{k}^{(3)}\right\} .
\end{aligned}
$$

Here, $\mathrm{p}_{i}$ is the $i$ :th partition, and $W_{j}^{i}$ is the $j$ :th cell of partition $i$. In the remainder of the paper, to keep notation uncluttered, we suppress indexes $i$ and $j$ for the partitions and cells.

It is quickly realised that as the size of the measurement set increases, the number of possible partitions grows very large. Thus, in order to have a computationally tractable target tracking method, only a subset of all possible partitions can be considered. We propose a simple heuristic for finding this subset of partitions, which is based on the distances between the measurements.

Given a set of measurements $\mathbf{Z}$ and distance thresholds $\left\{d_{i}\right\}_{i=1}^{N_{d}}$, with $d_{i}<d_{i+1}, \forall i$, for each $d_{i}$ we compute partitions where the cells constitute sets of measurements that are no more than $d_{i}$ metres apart from their closest cell neighbour. Thus, we get $N_{d}$ partitions, and since the $d_{i}$ are increasing, each partition contains fewer cells, and the cells typically contain more measurements.

The underlying intuition behind this idea is that two measurements generated by the same target are likely to be "close" to each other, while two measurements generated by different targets are likely to be "distant" from each other.
For two measurements $\mathbf{z}_{k}^{(1)}$ and $\mathbf{z}_{k}^{(2)}$, both measured with covariance $R_{k}=\sigma_{e}^{2} \mathbf{I}_{2}$, where $\mathbf{I}_{2}$ is a $2 \times 2$ identity matrix, the quantity

$$
\left(\mathbf{z}_{k}^{(1)}-\mathbf{z}_{k}^{(2)}\right)^{\mathrm{T}} R_{k}^{-1}\left(\mathbf{z}_{k}^{(1)}-\mathbf{z}_{k}^{(2)}\right)
$$

is $\chi^{2}$ distributed with 2 degrees of freedom. Equation (6) is the Mahalanobis distance between the two measurements, and can be seen as a measure of how "close" they are. Using the inverse $\chi^{2}$ distribution, a unit-less distance threshold $\delta_{P_{G}}$ can be computed for a given probability $P_{G}$. The test for whether $\mathbf{z}_{k}^{(1)}$ and $\mathbf{z}_{k}^{(2)}$ are "close" becomes

$$
\left(\mathbf{z}_{k}^{(1)}-\mathbf{z}_{k}^{(2)}\right)^{\mathrm{T}} R_{k}^{-1}\left(\mathbf{z}_{k}^{(1)}-\mathbf{z}_{k}^{(2)}\right)<\delta_{P_{G}} .
$$

Since $R_{k}^{-1}=\sigma_{e}^{-2} \mathbf{I}_{2}$ this inequality reduces to

$$
\left\|\mathbf{z}_{k}^{(1)}-\mathbf{z}_{k}^{(2)}\right\|_{2}<\sigma_{e} \sqrt{\delta_{P_{G}}} .
$$

Thus, for different probability levels $P_{G}$, different euclidean distance thresholds $\sigma_{e} \sqrt{\delta_{P_{G}}}$ can be computed.

Now, for a measurement set $\mathbf{Z}$, let $\left\{d_{i}^{m}\right\}_{i=1}^{N_{d}}$ be the set of unique measurement to measurement distances, sorted such that $d_{i}^{m}<d_{i+1}^{m}, \forall i$. In this work, we have found that using distance thresholds $d_{i}$ that correspond to the $d_{i}^{m}$ that are larger than $\sigma_{e} \sqrt{\delta_{0.30}}$ and smaller than $\sigma_{e} \sqrt{\delta_{0.80}}$ produces good sets of partitions.

An obvious problem with this approach occurs when two, or more, targets are close to each other. The generated measurements will also be close, and are thus likely to be deemed to belong to the same cell. In Section 5, where we present our target tracking results, we see that this is indeed a problem. It results in the underestimation of the number of existing targets. It remains within future work to find a better way to compute a suitable subset of all possible partitions

\section{The PHD Filter for Extended Targets}

The PHD prediction equations for extended targets are identical to those for single-measurement targets (targets that generate at most one measurement), hence those equations are not repeated here. Refer to [5] for the equations, and to [7] for the GM-PHD-filter implementation.

In the GM-PHD-filter by [7], the PHD-intensity is represented by a Gaussian mixture of the form

$$
v_{k}(\mathbf{x})=\sum_{i=1}^{J_{k}} w_{k}^{(i)} \mathcal{N}\left(\mathbf{x} \mid m_{k}^{(i)}, P_{k}^{(i)}\right),
$$

where $w_{k}^{(i)}, m_{k}^{(i)}$ and $P_{k}^{(i)}$ are the weights, mean vectors and covariance matrices of the Gaussian components, respectively. Note that the weights $w_{k}^{(i)}$ need not sum 
to one, i.e. it is not a probability distribution. When estimated target locations are needed, they are extracted the same way as is done in [7]. An estimate of the number of targets can be obtained by either computing the sum of the weights, or by extracting targets and counting the number of extractions.

In the single-measurement GM-PHD-filter measurement update, each measurement is used to update each Gaussian component. In its extended target equivalent, each cell of each partition is used to update each Gaussian component. Both these measurement updates increase the number of Gaussian components. To maintain a reasonable number of components $J_{k \mid k}$, in order to keep computations tractable, pruning and merging can be performed. For our extended target tracking, we use the same pruning and merging as presented in [7].

The PHD measurement update equations for the extended target Poisson model of [2] was derived in [6]. In Section 4.1 we present the measurement pseudolikelihood function from [6], and in Section 4.2 we present a Gaussian Mixture implementation of the PHD measurement update based on this measurement pseudo-likelihood function. In [5] Mahler derives a slightly more rigorous measurement likelihood function for extended targets which generate geometrically structured measurements, however this work will be limited to investigation of the Poisson model measurement likelihood.

\subsection{Pseudo-Likelihood Function}

If $v_{k \mid k-1}(\mathbf{x} \mid \mathbf{Z})$ is the predicted PHD-intensity, the corrected PHD-intensity is

$$
v_{k \mid k}(\mathbf{x} \mid \mathbf{Z})=L_{\mathbf{Z}_{k}}(\mathbf{x}) v_{k \mid k-1}(\mathbf{x} \mid \mathbf{Z})
$$

where the measurement pseudo-likelihood function [6] is given by

$$
\begin{aligned}
L_{\mathbf{Z}_{k}}(\mathbf{x})= & -\left(1-e^{-\gamma(\mathbf{x})}\right) p_{D}(\mathbf{x})+e^{-\gamma(\mathbf{x})} p_{D}(\mathbf{x}) \times \\
& \times \sum_{\mathrm{p} \angle \mathbf{Z}_{k}} \omega_{\mathrm{p}} \sum_{W \in \mathrm{p}} \frac{\gamma(\mathbf{x})^{|W|}}{d_{W}} \cdot \prod_{\mathbf{z} \in W} \frac{\phi_{\mathbf{z}}(\mathbf{x})}{\lambda_{k} c_{k}(\mathbf{z})}
\end{aligned}
$$

The first part of this equation, $1-\left(1-e^{-\gamma(\mathbf{x})}\right) p_{D}(\mathbf{x})$, handles the targets for which there are no detections. The second part handles targets for which there are at least one detection.

Here, the notation $\mathrm{p} \angle \mathbf{Z}_{k}$ means that $\mathrm{p}$ partitions the measurement set $\mathbf{Z}_{k}$ into cells $W$. The first summation is taken over all partitions $\mathrm{p}$ of the measurement set $\mathbf{Z}_{k}$. The second summation is taken over all cells $W$ in the current partition p, and the product is over all measurements $\mathbf{z}$ in the cell $W$. For each partition, the measurements in cells containing more than one measurement can be interpreted as coming from the same target. Measurements in cells with just one measurement can be either clutter or target generated. Here

$$
\omega_{\mathrm{p}}=\frac{\prod_{W \in \mathrm{p}} d_{W}}{\sum_{\mathrm{p}^{\prime} \angle \mathbf{Z}^{\prime}} \prod_{W^{\prime} \in \mathrm{p}^{\prime}} d_{W^{\prime}}}
$$

can be interpreted as a weight of the particular partion. Further

$$
\begin{aligned}
& d_{W}=\delta_{|W|, 1}+ \\
& v_{k \mid k-1}\left[e^{-\gamma(\mathbf{x})} \gamma(\mathbf{x})^{|W|} p_{D}(\mathbf{x}) \prod_{\mathbf{z} \in W} \frac{\phi_{\mathbf{z}}(\mathbf{x})}{\lambda_{k} c_{k}(\mathbf{z})}\right]
\end{aligned}
$$

where $\delta_{i, j}$ is the Kronecker delta and $|W|$ is the number of elements in $W$. For any function $h(\mathbf{x})$,

$$
v_{k \mid k-1}[h]=\int h(\mathbf{x}) v_{k \mid k-1}(\mathbf{x} \mid \mathbf{Z}) d \mathbf{x} .
$$

The expected number of generated measurements is denoted $\gamma(\mathbf{x})$, and the probability of getting at least one detection is

$$
1-e^{-\gamma(\mathbf{x})}
$$

With the probability of detection $p_{D}(\mathbf{x})$ the effective probability of detection becomes

$$
\left(1-e^{-\gamma(\mathbf{x})}\right) p_{D}(\mathbf{x})
$$

The spatial distribution of the measurements are described by the function

$$
\phi_{\mathbf{z}}(\mathbf{x})=\phi(\mathbf{z} \mid \mathbf{x})
$$

and the clutter distribution is modelled by $\lambda_{k} c_{k}(\mathbf{z})$. Here, $\lambda_{k}$ is the Poisson rate that determines the number of clutter per scan, and $c_{k}(\mathbf{z})$ is the spatial distribution of the clutter measurements.

\subsection{Gaussian Mixture Implementation}

Following the derivation of a GM-PHD-filter for single measurement targets in [7], a PHD recursion can be derived for the extended target case. Here, we have made the same six assumptions that are made in [7]. Further, we make the following assumption:

Assumption: The expected number of generated measurements $\gamma(\mathbf{x})$ can be approximated as functions of the mean of the individual Gaussian components $\gamma^{(j)} \triangleq \gamma\left(m_{k \mid k-1}^{(j)}\right)$.

Remark: The assumption is resonable, since the number of generated measurements is largely decided by the target's spatial extent. The spatial extent can be modelled using parameters which are included in the target state vector $[1-3,8]$, thus the number of generated measurements can be seen as a function of the predicted mean of the Gaussian component. 
The measurement updated PHD intensity is then as follows. Let the predicted intensity be a Gaussian mixture of the form

$$
v_{k \mid k-1}(\mathbf{x})=\sum_{i=1}^{J_{k \mid k-1}} w_{k \mid k-1}^{(i)} \mathcal{N}\left(\mathbf{x} \mid m_{k \mid k-1}^{(i)}, P_{k \mid k-1}^{(i)}\right) .
$$

Then, the posterior intensity at time $k$ is a Gaussian mixture given by

$$
v_{k \mid k}(\mathbf{x})=v_{k \mid k}^{N D}(\mathbf{x})+\sum_{\mathrm{p} \angle \mathbf{Z}^{\prime}} \sum_{W \in \mathrm{p}} v_{k \mid k}^{D}(\mathbf{x}, W),
$$

where the Gaussian components handling no detections are given by

$$
\begin{aligned}
v_{k \mid k}^{N D}(\mathbf{x}) & =\sum_{j=1}^{J_{k \mid k-1}} w_{k \mid k}^{(j)} \mathcal{N}\left(\mathbf{x} \mid m_{k \mid k}^{(j)}, P_{k \mid k}^{(j)}\right) \\
w_{k \mid k}^{(j)} & =\left(1-\left(1-e^{-\gamma^{(j)}}\right) p_{D}\right) w_{k \mid k-1}^{(j)}, \\
m_{k \mid k}^{(j)} & =m_{k \mid k-1}^{(j)} \\
P_{k \mid k}^{(j)} & =P_{k \mid k-1}^{(j)}
\end{aligned}
$$

and the Gaussian components handling detected targets are given by

$$
\begin{aligned}
v_{k \mid k}^{D}(\mathbf{x}, W) & =\sum_{j=1}^{J_{k \mid k-1}} w_{k \mid k}^{(j)} \mathcal{N}\left(\mathbf{x} \mid m_{k \mid k}^{(j)}, P_{k \mid k}^{(j)}\right), \\
w_{k \mid k}^{(j)} & =\omega_{\mathrm{p}} \frac{\Gamma^{(j)} p_{D}}{d_{W}} \Phi_{W}^{(j)} w_{k \mid k-1}^{(j)}, \\
\Gamma^{(j)} & =e^{-\gamma^{(j)}}\left(\gamma^{(j)}\right)^{|W|}, \\
\Phi_{W}^{(j)} & =\prod_{\mathbf{z} \in W} \frac{\phi_{\mathbf{z}}\left(m_{k \mid k-1}^{(j)}\right)}{\lambda_{k} c_{k}(\mathbf{z})},
\end{aligned}
$$

and the probability of the measurements in cell $W$ is

$$
\phi_{\mathbf{z}}\left(m_{k \mid k-1}^{(j)}\right)=\mathcal{N}\left(\mathbf{z} \mid H_{k} m_{k \mid k-1}^{(j)}, R_{k}+H_{k} P_{k \mid k-1}^{(j)} H_{k}^{\mathrm{T}}\right) .
$$

The partition weights $\omega_{\mathrm{p}}$ are given by

$$
\begin{aligned}
\omega_{\mathrm{p}} & =\frac{\prod_{W \in \mathrm{p}} d_{W}}{\sum_{\mathrm{p}^{\prime} \angle \mathbf{Z}^{\prime}} \prod_{W^{\prime} \in \mathrm{p}^{\prime}} d_{W^{\prime}}}, \\
d_{W} & =\delta_{|W|, 1}+\sum_{l=1}^{J_{k \mid k-1}} \Gamma^{(l)} p_{D} \Phi_{W}^{(l)} w_{k \mid k-1}^{(l)} .
\end{aligned}
$$

The mean and covariance of the Gaussian components are updated using the standard Kalman measurement update,

$$
\begin{aligned}
m_{k \mid k}^{(j)} & =m_{k \mid k-1}^{(j)}+\mathbf{K}_{k}^{(j)}\left(\left[\begin{array}{c}
\mathbf{z}_{1} \\
\vdots \\
\mathbf{z}_{|W|}
\end{array}\right]-\mathbf{H}_{k} m_{k \mid k-1}^{(j)}\right) \\
P_{k \mid k}^{(j)} & =\left(I-\mathbf{K}_{k}^{(j)} \mathbf{H}_{k}\right) P_{k \mid k-1}^{(j)} \\
\mathbf{K}_{k}^{(j)} & =P_{k \mid k-1}^{(j)} \mathbf{H}_{k}^{\mathrm{T}}\left(\mathbf{H}_{k} P_{k \mid k-1}^{(j)} \mathbf{H}_{k}^{\mathrm{T}}+\mathbf{R}_{k}\right)^{-1}
\end{aligned}
$$

Here, if the current cell $W$ contains $|W|=3$ measurements,

$$
\begin{aligned}
\mathbf{H}_{k} & =\left[\begin{array}{c}
H_{k} \\
H_{k} \\
H_{k}
\end{array}\right], \\
\mathbf{R}_{k} & =\operatorname{blkdiag}\left(R_{k}, R_{k}, R_{k}\right) .
\end{aligned}
$$

\section{Simulation Results}

This section presents results from simulations using the presented extended target tracking method. Section 5.1 presents the simulation setup, and the following subsections presents simulation results using our method compared to results using standard GM-PHD. The MATLAB code used to produce the simulations and figures is available online ${ }^{1}$.

\subsection{Simulation Setup}

The targets' centers of mass are modelled as points with states variables

$$
\mathbf{x}_{k}=\left[\begin{array}{llll}
\mathrm{x}_{k} & \mathrm{y}_{k} & v_{k}^{\times} & v_{k}^{\mathrm{y}}
\end{array}\right]^{\mathrm{T}},
$$

where $\mathrm{x}_{k}, \mathrm{y}_{k}$ is the planar position of the target in meters, and $v_{k}^{\mathrm{x}}$ and $v_{k}^{\mathrm{y}}$ are the corresponding velocities in meters per second. The sensor measurements are given in batches of Cartesian $x$ and $y$ coordinates as follows;

$$
\mathbf{z}_{k}^{(j)} \triangleq\left[\begin{array}{ll}
\mathbf{x}_{k}^{(j)} & \mathbf{y}_{k}^{(j)}
\end{array}\right]^{\mathrm{T}} .
$$

In many real world applications (e.g. radar, laser and stereo vision), the sensor measures range $r$ and azimuth angle $\varphi$ given as

$$
\overline{\mathbf{z}}_{k}^{(j)} \triangleq\left[\begin{array}{ll}
r_{k}^{(j)} & \varphi_{k}^{(j)}
\end{array}\right]^{\mathrm{T}}
$$

The work here could be extended to such a case using the appropriate polar to Cartesian conversion equations, in order to convert the measurements into the

\footnotetext{
${ }^{1}$ http://www . control. isy. liu.se/publications/doc?id=2299 Contact the authors if the link is broken.
} 

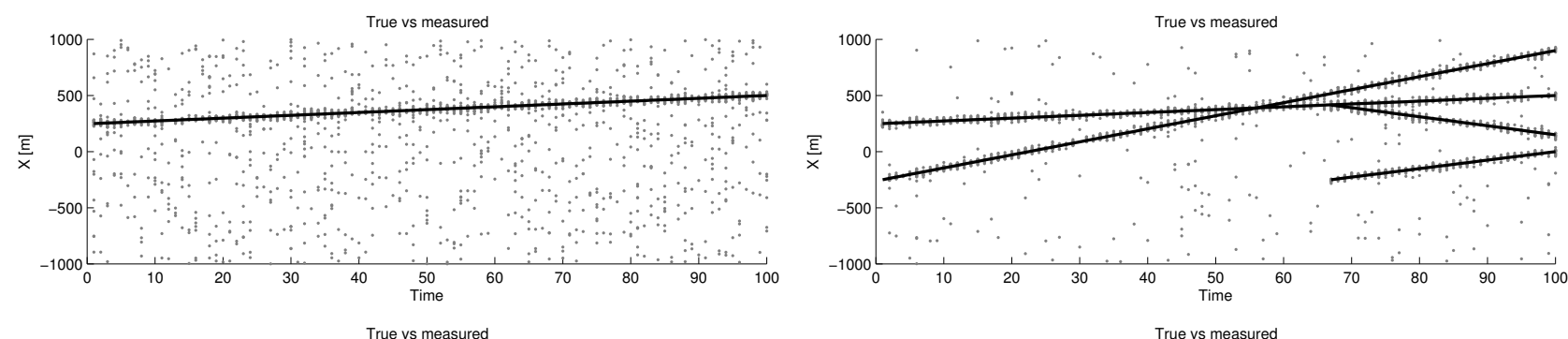

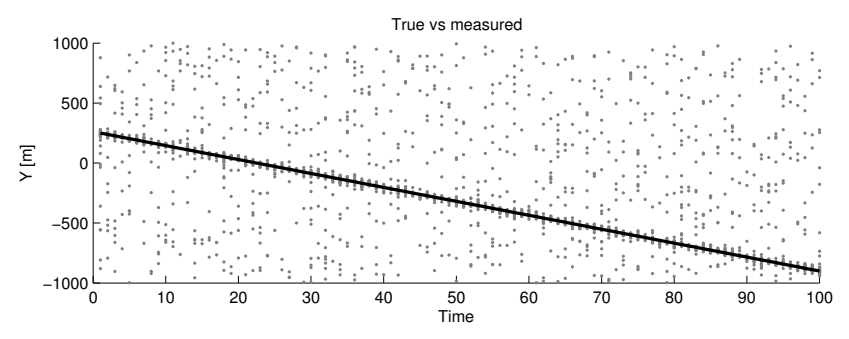

(a) One target present

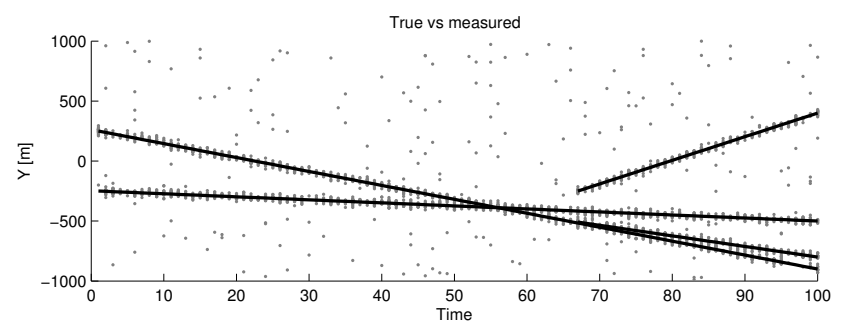

(b) Multiple targets present

Figure 3: The true $\mathrm{x}$ and $\mathrm{y}$ positions in black, and measurements in gray.

form (25). Using the following dynamic and measurement models,

$$
\begin{aligned}
F_{k} & =\left[\begin{array}{llll}
1 & 0 & T & 0 \\
0 & 1 & 0 & T \\
0 & 0 & 1 & 0 \\
0 & 0 & 0 & 1
\end{array}\right], G_{k}=\left[\begin{array}{cc}
\frac{T^{2}}{2} & 0 \\
0 & \frac{T^{2}}{2} \\
T & 0 \\
0 & T
\end{array}\right], \\
H_{k} & =\left[\begin{array}{llll}
1 & 0 & 0 & 0 \\
0 & 1 & 0 & 0
\end{array}\right], Q_{k}=\sigma_{\mathbf{w}}^{2} \mathbf{I}_{2}, R_{k}=\sigma_{\mathbf{e}}^{2} \mathbf{I}_{2},
\end{aligned}
$$

with sampling time $T=1$ s and the implemented extended target measurement likelihood, target tracking was performed on simulated data. Here, $\sigma_{\mathbf{w}}=2 \mathrm{~m} / \mathrm{s}^{2}$ and $\sigma_{\mathbf{e}}=20 \mathrm{~m}$. The presented tracking filter was first tested in a scenario with one target present. The filter was then tested in a scenario with multiple targets, target spawning and birth of new targets. In each simulation, there was also clutter measurements.

For both simulations, the surveillance area is $[-1000 \mathrm{~m}, 1000 \mathrm{~m}] \times[-1000 \mathrm{~m}, 1000 \mathrm{~m}]$. The probability of survival is set to $p_{S}=0.99$, and the probability of detection is $p_{D}=0.99$. The Poisson rate for the number of measurements generated per time step is set to 10 for each target, i.e. $\gamma^{(i)}=10, \forall i$.

The birth intensity is

$$
\begin{aligned}
v_{b}(\mathbf{x}) & =0.1 \mathcal{N}\left(\mathbf{x} ; m_{b}^{(1)}, P_{b}\right)+0.1 \mathcal{N}\left(\mathbf{x} ; m_{b}^{(2)}, P_{b}\right), \\
m_{b}^{(1)} & =[250,250,0,0]^{\mathrm{T}}, \\
m_{b}^{(2)} & =[-250,-250,0,0]^{\mathrm{T}}, \\
P_{b} & =\operatorname{diag}([100,100,25,25]) .
\end{aligned}
$$

The spawn intensity is

$$
v_{\beta}(\mathbf{x} \mid \mathbf{y})=0.05 \mathcal{N}\left(\mathbf{x} ; \xi, Q_{\beta}\right),
$$

where $Q_{\beta}=\operatorname{diag}([100,100,400,400])$, and $\xi$ is the target from which the new target is spawned. This example is very similar to the one presented in [7].

\subsection{Single target}

Figure 3 shows the cluttered measurement set used in the simulation. The target extractions resulting from extended target and single-measurement GM-PHD filtering are compared to ground truth in Figures 4 and 5 . The estimated number of targets is compared to ground truth in Figures 6 and 7, from which it is obvious that the suggested extended target GM-PHD filter outperforms the standard singe-measurement GM-PHD. In terms of the locations of the extracted targets, the extended target filter is again better than the singlemeasurement filter. Using the presented extended target tracking method in a single target scenario, the right number of targets is found, and the target location is correctly estimated.

\subsection{Multiple targets}

The same comparison of tracking methods was performed using simulated data for four targets. For this data, shown in Figure 8, two of the true target tracks cross at time $k=56$, and one of the target tracks is spawned at time $k=66$. The results are shown in Figure ??. Again, it is apparent that the number of targets is overestimated when single-measurement techniques are used, see Figure 12. Tracking performance is much better when the suggested extended target tracking method is used.

Further, in Figure 11 it can be seen that the number of targets are not estimated correctly when two, or more, targets are close to each other, i.e. when tracks cross or when new targets are spawned. One potential 

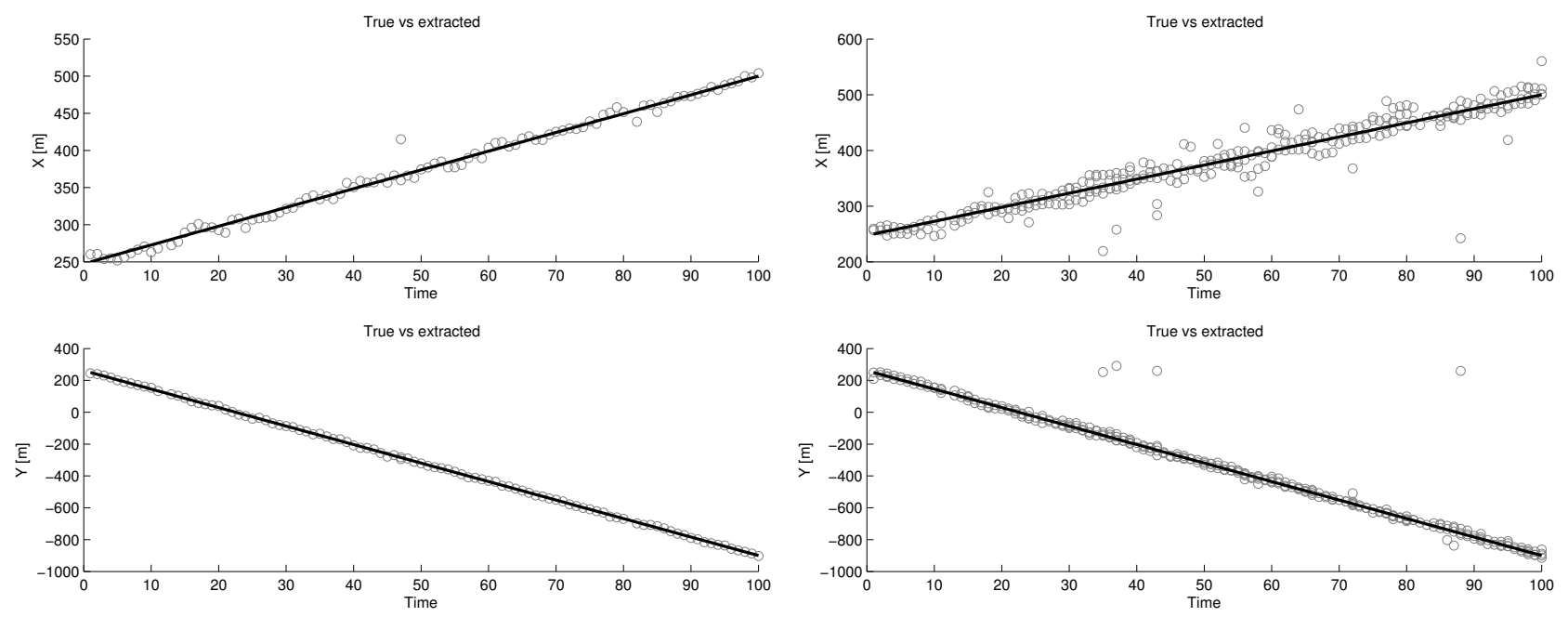

(a) Our extended target GM-PHD

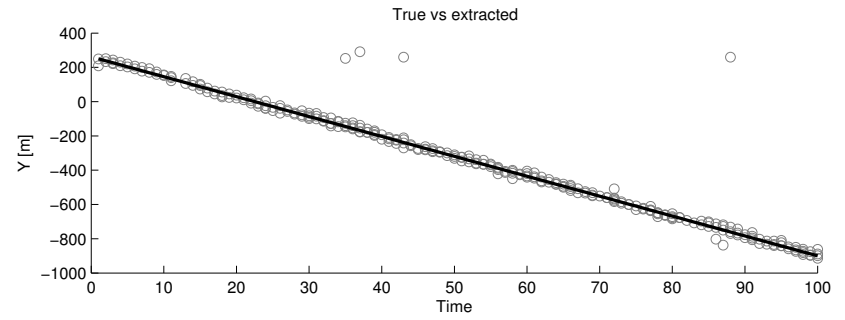

(b) Single-measurement GM-PHD [7]

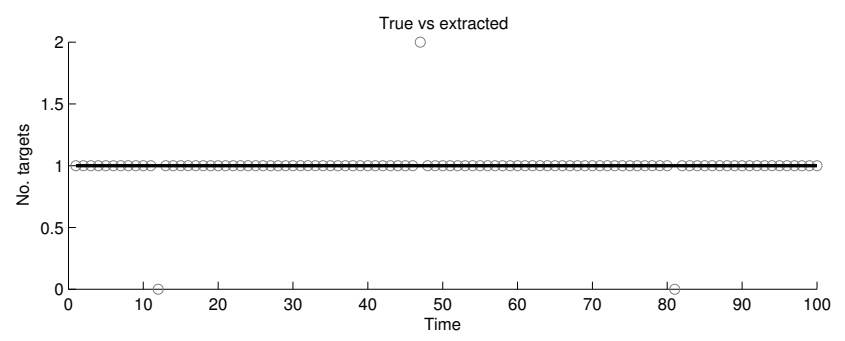

(c) Our extended target GM-PHD

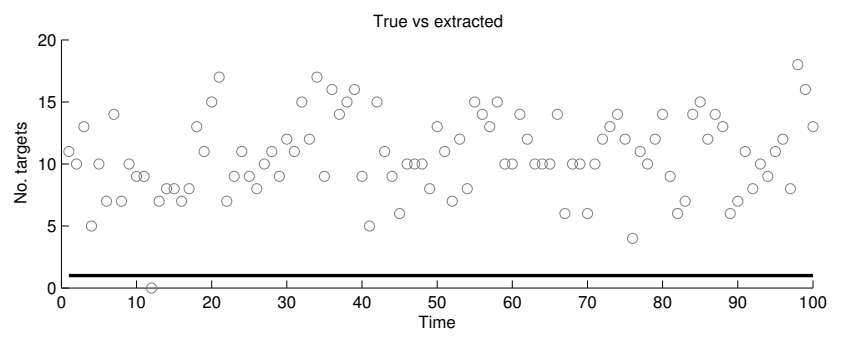

(d) Single-measurement GM-PHD [7]

Figure 4: Target tracking results - one target present. In (a) and (b), the true $\mathrm{x}$ and $\mathrm{y}$ positions are in black, and the $\mathrm{x}$ and $\mathrm{y}$ position of the extracted GM-PHD components are in gray. In (c) and (d), the black line is the true number of targets, and gray rings are the extracted number of targets. Despite the high number of clutter measurements, using our extended target GM-PHD there is only one false extracted track at time $k=47$.

reason for this is the rather naive approach to finding a good set of partitions for the measurement set. When the set of measurements is partitioned using different distances, measurements from two closely spaced targets will also be closely spaced, and thus belong to the same cell in the partition. To resolve this issue, a more complex method for partitioning the measurement set has to be used. Despite the underestimation of number of targets, the estimation of target location remains good.

\section{Conclusions and Future Work}

In this paper we have presented a Gaussian mixture probability hypothesis density filter for tracking of extended targets. Further, we have presented a simple heuristic for finding a subset of all measurement set partitions. With simulations, we have shown that our filter is capable of tracking extended targets in cluttered measurements. The number of targets is estimated correctly, with the exception of when tracks cross or new targets spawn from existing targets.

In future work, we plan on investigating the possi- bility of finding a better method for measurement set partitioning. If the Poisson rate of the measurement generating process is known, it could possibly be used to find good partitions.

We also plan to use our GM-PHD-filter to track targets which generate measurements that are geometrically structured, and try to infer the spatial extension or shape from these measurements. Work is underway that tracks vehicles using laser range sensors. Under the assumption that vehicles are rectangular in shape, in preliminary results we are able to track not only position and heading of the vehicles, but also the width and length. These results are at the time of writing not ready for publication.

\section{Acknowledgments}

The authors would like to thank the Linnaeus research environment CADICS, funded by the Swedish Research Council and the Strategic Research Center MOVIII, funded by the Swedish Foundation for Strategic Research, for financial support. 

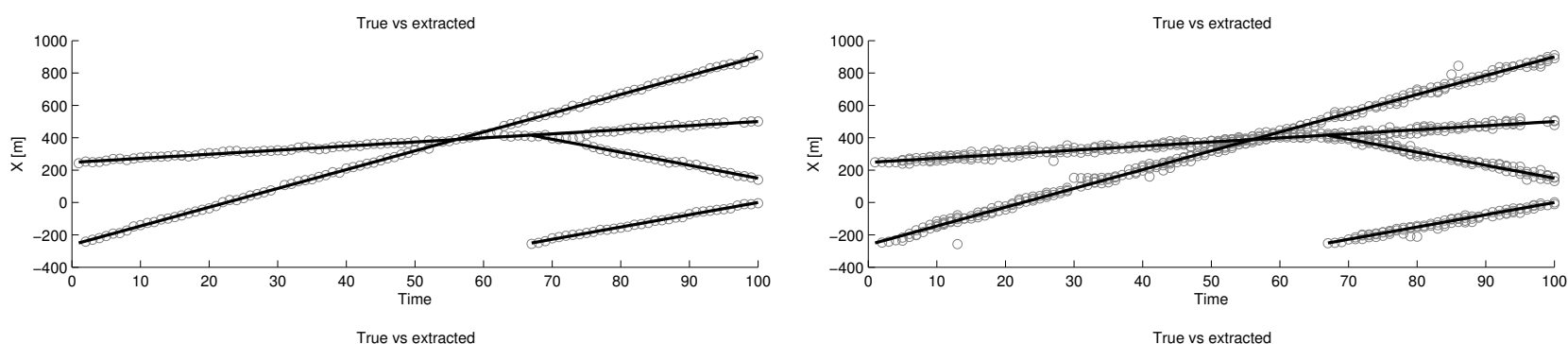

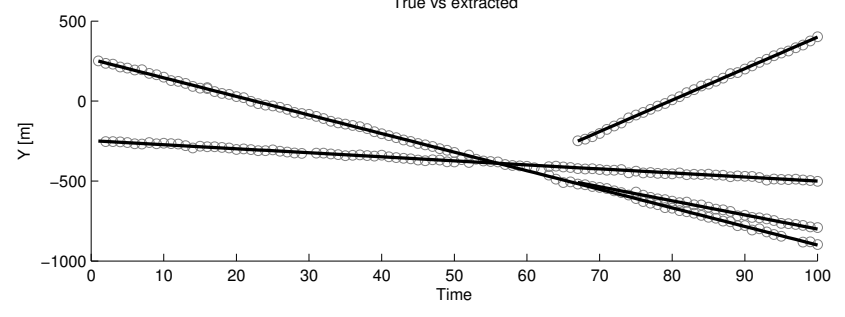

(a) Our extended target GM-PHD

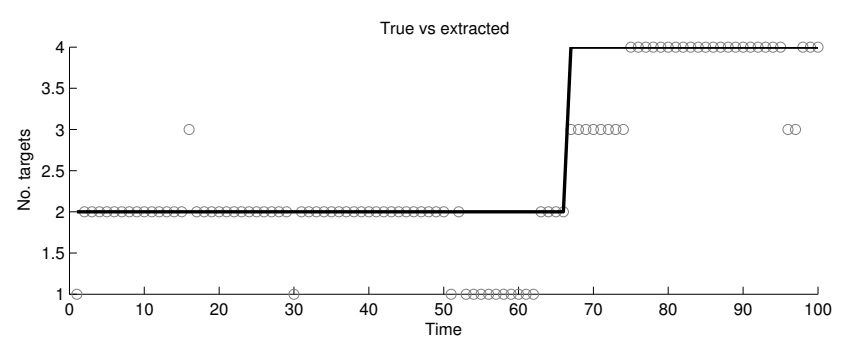

(c) Our extended target GM-PHD

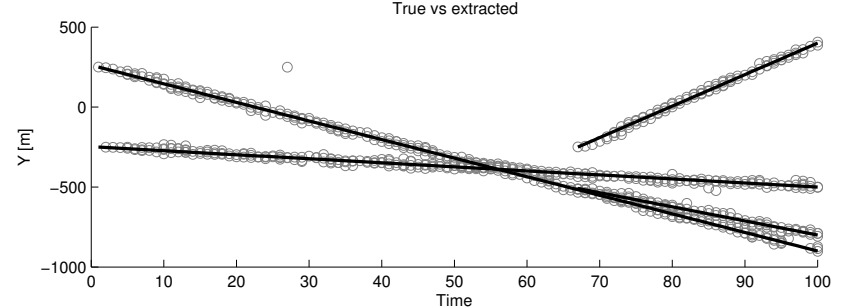

(b) Single-measurement GM-PHD [7]

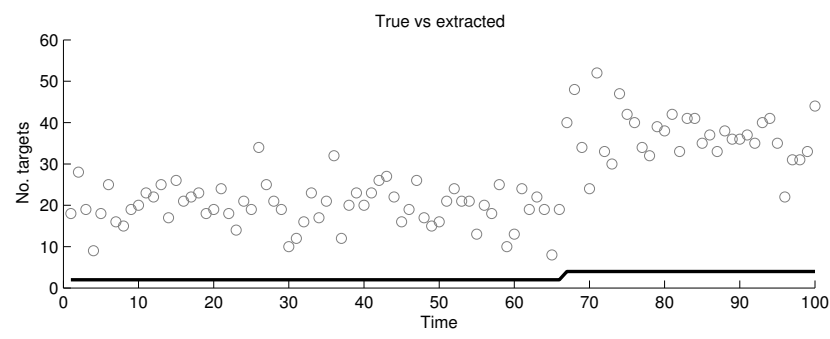

(d) Single-measurement GM-PHD [7]

Figure 5: Target tracking results - multiple targets present. In (a) and (b), the true $\mathrm{x}$ and $\mathrm{y}$ positions are in black, and the $x$ and $y$ position of the extracted GM-PHD components are in gray. In (c) and (d), the black line is the true number of targets, and gray rings are the extracted number of targets.

\section{References}

[1] Y. Boers, H. Driessen, J. Torstensson, M. Trieb, R. Karlsson, and F. Gustafsson. A track before detect algorithm for tracking extended targets. IEE Proceedings Radar, Sonar and Navigation, 153(4):345-351, August 2006.

[2] K. Gilholm, S. Godsill, S. Maskell, and D. Salmond. Poisson models for extended target and group tracking. In Proceedings of Signal and Data Processing of Small Targets, volume 5913, pages 230-241, San Diego, CA, USA, August 2005. SPIE.

[3] K. Gilholm and D. Salmond. Spatial distribution model for tracking extended objects. IEE Proceedings Radar, Sonar and Navigation, 152(5):364-371, October 2005.

[4] R.P.S. Mahler. Multitarget Bayes filtering via firstorder multi target moments. IEEE Transactions on Aerospace and Electronic Systems, 39(4):1152-1178, October 2003.
[5] R.P.S. Mahler. Statistical Multisource-Multitarget Information Fusion. Artech House, Inc., Norwood, MA, USA, 2007.

[6] R.P.S. Mahler. PHD filters for nonstandard targets, I: extended targets. In Proceedings of the 12th International Conference on Information Fusion, pages 915-921, Seattle, WA, USA, July 2009.

[7] B.-N. Vo and W.-K. Ma. The Gaussian mixture probability hypothesis density filter. IEEE Transactions on Signal Processing, 54(11):40914104, November 2006.

[8] Z. Zhong, H. Meng, and X. Wang. Extended target tracking using an IMM based Rao-Blackwellised unscented Kalman filter. In Proceedings of 9th International Conference on Signal Processing ICSP, pages 2409-2412, October 2008. 